\section{Imaging a Biological Sample Using a Scanning Probe Microscope}

Briggs Christie, TopoMetrix

Scanning Probe Microscopy (SPM) has seen limited use in the field of biotechnology until recently due to problems arising from:

$\checkmark$ Scanning under adverse conditions.

$\checkmark$ Precise tip positioning.

$\checkmark$ Extensive sample damage due to multiple scans.

The success of experiments such as the one described in this article have lead to the development of SPM instruments that address these and other issues, bringing nanoscale 3 -dimensional imaging to the biological researcher.

In this experiment, fluorescently labeled polythene chromosomes acquired from a Drosophila salivary gland were absorbed to a glass slide. The slide was placed on a Zeiss Axiovert ${ }^{\text {Th }} 35$ inverted light microscope and the AFM was placed above the sample with the tip positioned in the center of the optical field (See Figure 1).

The inverted light microscope was used to position the AFM tip over a small cluster of chromosomes for AFM scanning. Due to the liquid environment, meniscus forces were eliminated and imaging could be done at much lower forces than when performed in ambient air

A standard pyramidal AFM cantilever (200 micron cantilever arms, spring constant of $.03 \mathrm{~N} / \mathrm{m}$ ) was used. As shown in Figure 2, the scanner configuration allowed simultaneous viewing of fully hydrated polythene chromosomes in the light microscope while imaging with the AFM without the use of an external fluid cell. Blue attenuator filters were used and all operations were performed at room temperature.

The light microscope allowed extremely accurate positioning of the AFM tip over the sample, which was then scanned in "contact" mode. A comparison of the light microscope image and AFM image in Figure 3 shows the data acquired using this technique. The chromosome bands and the fine structure of the interbands are clearly visible in three dimensions. Due to the unique abilities of the life sciences configuration of the TopoMetrix TMX 2000 Explorer ${ }^{\text {Th }}$ SPM, lateral force, modulated force and non-contact images could be simultaneously obtained in a single scan.

The use of an inverted light microscope for sample location brings the AFM into the biotechnology laboratory, offering the entire continuum of tip modulation scanning techniques as well as conventional contact and lateral force imaging to the researcher. The ability to image under liquid allows AFM imaging of biological samples in a natural environment and allows the use of light forces, minimizing sample distortion.

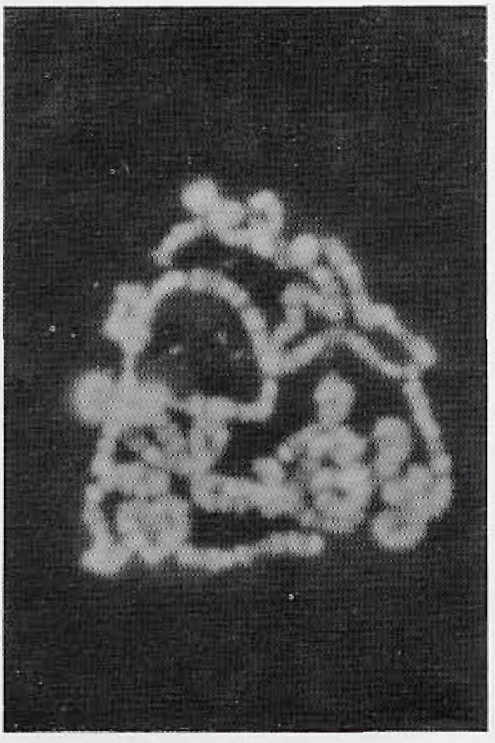

Floursced Light Microscope Image

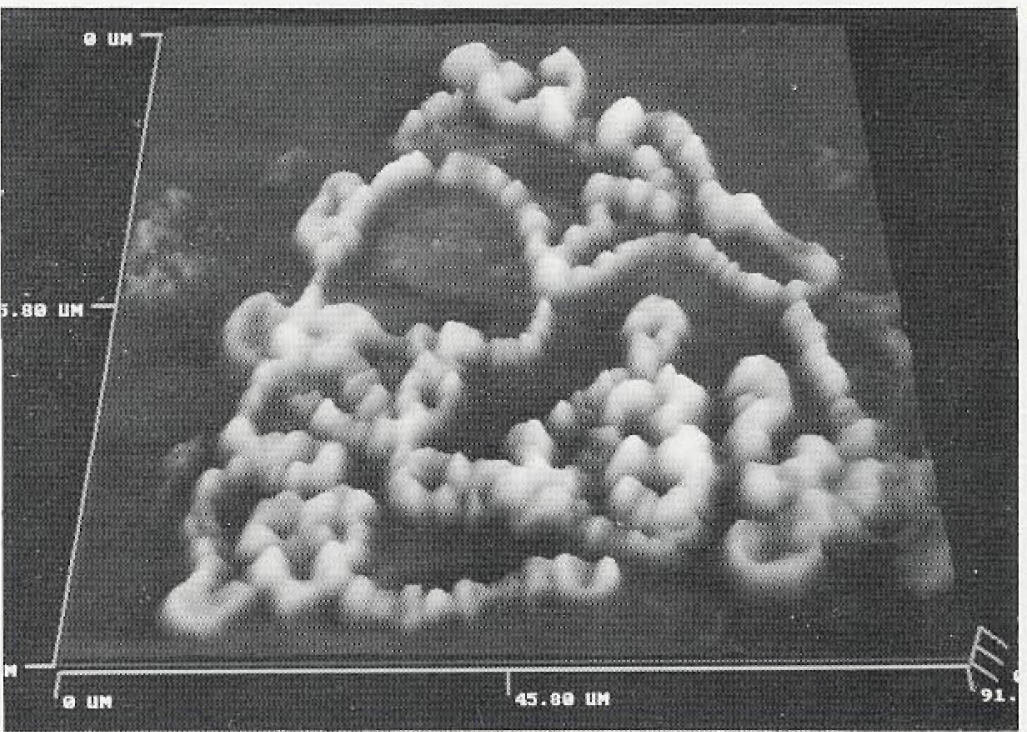

AFM Image 


\section{$\downarrow \downarrow$ Note New Advertising Rates: \$50 for the first line and \$25 for each additional line}

Electron Microscopist to provide technical support to multi-user/multidisciplinary core research facility. Responsibilities include operation of SEMs and TEMs and all aspects of preparation of materials and biological samples. Film development and printing, preparation of solutions, record keeping, supply inventory/ordering and other general laboratory skills will be required. While training/experience in EM is required, a B.Sc. or M.Sc. in Biology, Chemistry or related field, and MSA certification, are desirable. Contact Carol S. Williams, Director, Electron Microscopy Services, Carver Research Laboratory, Rm 2, Tuskegee University, Tuskegee, AL 36088.

Thomas Jefferson University, Philadelphia, PA, is seeking an experienced research electron microscopist. This individual will study the ultrastructure of peptide nerve terminals in the medulla of the rat. For more information, contact Dr. Richard Lynn at (215)955-4152 or -6944.

HITACHI S-2400 SEM, 2 years old. With Kevex Level 1 EDS (including X-ray mode \& Superquantum, and Quantum X-Rray detector), Robinson BSE detector, raster rotation, graphics generator, printer and chiller system. Granmont, Inc. Contact Jim Steinnecker, Tel.: (602)889-5424

PHILIPS 300 TEM for sale, excellent condition, under contract 'til last year. Best Offer. Contact EMSI, Peter Jordan: (909)694-1839.

Several Ainsworth Analytical Balances. $160 \mathrm{~g}$ capacity with $1 \mathrm{mg}$ resolution. Excellent condition. Call (608)836-4543 and leave message.

HITACHI S-800 Field Emission SEM: 4 years old, includes Raith motorized stage, Hitachi critical dimension measurement system, and Link eXL light element EDS system. or HITACHI S-2700 LaB6 SEM: $31 / 2$ years old, includes motorized-insertion Robinson backscatter detector, Link eXL light element EDS system and LEMAS motorized stage. Contact John Tartaglia, Bandgap Technology Corporation, Tel.: (303)460-0700, Ext. 366.

FROM:

$\mathrm{Dr}, \mathrm{Ms}$

Company

Dept/MS/B/dg/etc.

Street

City State Zip

Telephone No.

Please see that I receive futher information on the products and services, as presented in your November issue, as follows:

A New Series of SPMs: Burleigh Instruments

B New TEMs \& SEMs: Carl Zeiss, Inc.

_C Cold Sputter/Etch Unit: Denton Vacuum

D Nanoscope - MultiMode SPM: Digital Instruments

E Mag., X-ray \& Surface Reference Stds: Geller MicroAnalytical

F New X-Ray Detector Calibration Stds: Geller MicroAnalytical

G High Precision Positioning Stage: Hessler Technical Services

- H Cameca Microanalysis Software: Hessler Technical Services

- New Model S-4500 FE-SEM: Hitachi Scientific

_J Electron Microscopy Supplies: National Graphic Supplies

K New Full Spectrum WDS for SEMs: Peak Instruments

L Unicryl Resin \& Staining Kits: SPI Supplies

M Microscopy Books: Springer-Verlag

N SPM - Experimeter's Tool Kit: Topometrix

O Diffraction Simulation \& Analysis Software: Virtual Laboratories

P Stopping Carbon Buildup: XEI Scientific
Readers who wish to continue to receive a no cast copy of this neusletter, and have not freviously done sa, are encouraged to complete the following. Thank you.

We would prcps-ty be considered a (check the most appropriate): _ A User of microscopy equipment/ materials/services.

_B Supplier of microscopy equipment/ materials/services.

Our interests include (check all applicable)

_C Physical/Material Sciences.

D Biological/Biomedical Sciences.

E Earth Sciences.

My own professional interests include (check all applicable)

F Electron Microscopy.

G Confocal Microscopy.

H SPM/AFM.

_I Light Microscopy.

_J IR Microscopy.

_K Acoustic Microscopy.

After completion. please detach along 'perf', fold and tale closed with scouth tape. Thante you! 